\title{
EL MAGISTERIO DEL PROFESOR D. FRANCISCO ROMERO AGUIRRE
}

\author{
Mariano Pérez Albacete y Francisco Romero Fernández?.
}

Hospital Universitario Virgen de la Arrixaca. Murcia y Hospital Clínico Universitario'. Zaragoza. España.

\begin{abstract}
Resumen.- OBJETIVO: Con ocasión del fallecimiento del profesor D. Francisco Romero Aguirre (Zaragoza, 7.IV. 1918-28.VI.2006) queremos poner de manifiesto su gran vocación docente, para lo que recorremos su vinculación a la Universidad desde el comienzo de sus estudios hasta su magisterio como primer catedrático de Urología de la Licenciatura en la Universidad española (1961), y su labor al frente de la Escuela Profesional de postgrado en Urología de la Universidad de Zaragoza.
\end{abstract}

MÉTODOS: Revisamos su biografía, su obra editada y las publicaciones de la Cátedra de Urología de la Universidad de Zaragoza y extraemos cuantas referencias encontramos de su actividad docente.

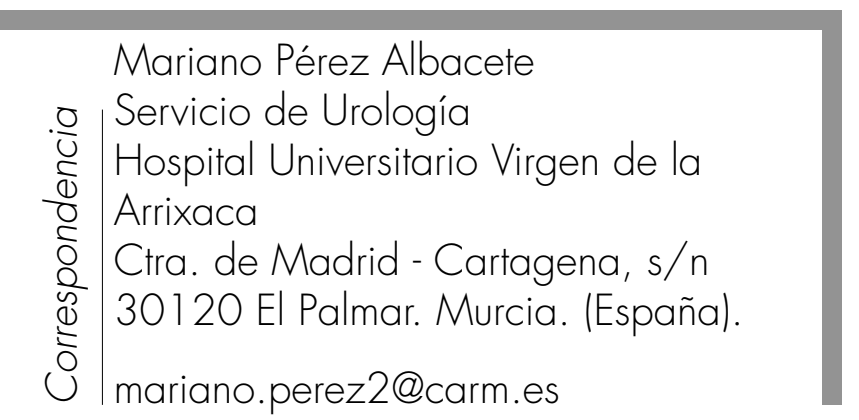

RESULTADO: Desde el inicio de su formación académica mantuvo una constante unión con la Universidad y colaboró en todas las disciplinas con las que tuvo relación hasta lograr ser responsable de la enseñanza de la Urología. Su talante universitario y su gran preparación hicieron ver al catedrático de Patología Quirúrgica la conveniencia de separar la Urología del tronco común de la Cirugía, propuesta que, aceptada por el claustro de la Facultad de Medicina de Zaragoza y elevada al ministerio, dio lugar a la designación de la Urología como asignatura independiente en la Licenciatura de Medicina. Su labor como responsable estuvo marcada por el trabajo y constantes logros en las clases, por numerosas publicaciones, por realización de cursos de doctorado y monográficos de Urología y por la dirección de tesis y memorias, todo lo cual permitieron la creación de la Escuela profesional de especialización urológica con la que culminaron sus más de cuarenta años de dedicación al magisterio universitario.

CONCLUSIÓN: Encontramos en el profesor Romero una vida dedicada íntegramente entregada a la Universidad y a su magisterio. Su gran formación urológica y propedéutica hicieron que fuese la persona que reunía las condiciones y las aptitudes necesarias para hacerse cargo y desempeñar satisfactoriamente el puesto, en la Facultad de Medicina de Zaragoza, de responsable de la enseñanza de la Urología, que, gracias a su virtud y buen hacer, fue elevada por vez primera en la Licenciatura española al rango de asignatura.

Palabras clave: Francisco Romero Aguirre. Docencia Urológica. Escuela profesional de Urología. Urología en la Universidad. Historia de la Urología. 
Summary.- OBJECTIVES: After the death of Professor Francisco Romero Aguirre (Zaragoza 6/7/1918$6 / 28 / 2006)$ we want to make known his great teaching vocation, so that we covered his link with University from the start of his studies to his professorship as first professor in urology in the Spanish University (1961), and his work at the chairmanship of the Professional Postgraduate School of Urology in the University of Zaragoza.

METHODS: We reviewed his biography, his edited works and publications of the Urology Chair at the University of Zaragoza, and obtained as many references as we found about his teaching activity.

RESULTS: From the start of his academic training he kept a constant union with University and cooperated in all disciplines he had relation with before he got the responsibility of teaching urology. His university spirit and great formation had the chairman of surgical pathology see the convenience of separating urology from the common trunk of surgery, a proposal that, being accepted by the staff meeting of the Faculty of Medicine of Zaragoza and submitted to the Ministry resulted in the designation of urology as an independent subject in the degree of medicine. His labour as responsible was marked by work and continuous achievements in his classes, many publications, doctorate and urological monographic courses carried out, thesis and memories directed, all of which enabled him to create to the Professional Urological Specialization School, the culmination of more than 40 years dedicated to teaching in the university.

CONCLUSIONS: We find Prof. Romero's life completely dedicated to University and teaching. His wide urological and propedeutical formation made him a person who joined the necessary conditions and abilities to take the responsibility and carry out a satisfactory performance as responsible of teaching urology in the school of medicine of Zaragoza, a subject which thanks to his virtue and good performance was for the first time elevated to the level of independent subject.

Keywords: Francisco Romero Aguirre. Urology teaching. Professional school of urology. Urology in the University. History of Urology.

\section{INTRODUCCIÓN}

Comenzó su formación universitaria, en 1933, tras su paso por el colegio de los P. P. Jesuitas de su ciudad natal; a lo largo de sus años de estudiante fue jefe de mesa de disección anatómica en la cátedra del profesor Guisande, recibió el encargo de

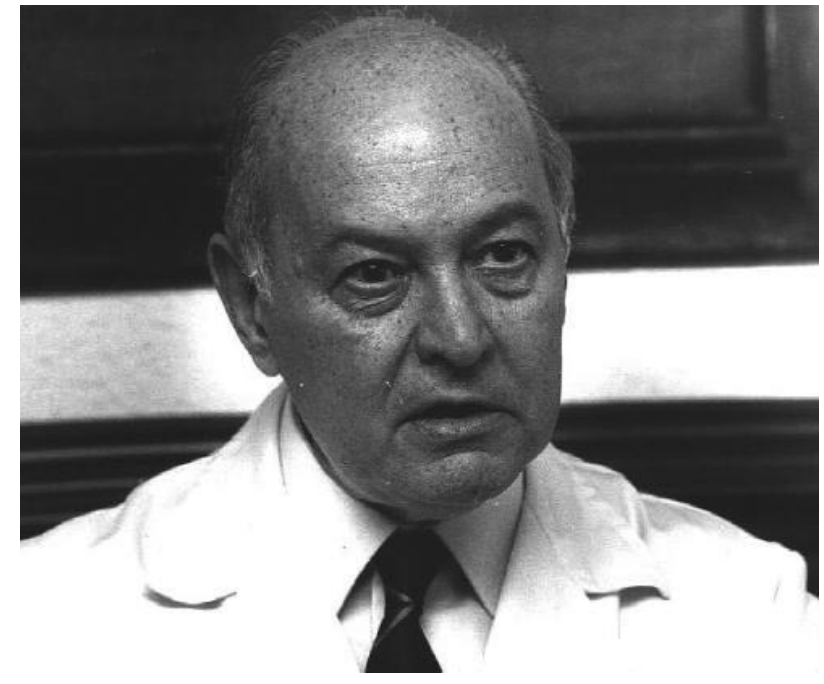

FIGURA 1. Profesor D. Francisco Romero Aguirre en 1988.

dibujar las preparaciones histológicas de las clases de Histología con el profesor Muniesa y colaboró en la cátedra de Farmacología con los Drs. Velázquez, primero, y Mateo Tinao, después; en los cursos clínicos fue alumno interno de Patología Quirúrgica con los profesores Sánchez Cozar y Lozano Blesa. Tras el forzoso paréntesis de la guerra civil, finalizó la licenciatura en 1941 con la calificación de sobresaliente (Figura 1).

Prosiguió con su vinculación a la Universidad y, entre 1943 y 1946, fue nombrado profesor de clases prácticas de Farmacología Clínica. Elaboró la memoria para obtener el grado de doctor en Madrid, en la cátedra de Terapéutica Quirúrgica de la Universidad Central, bajo la dirección del profesor Estella, versó sobre Hipertensión arterial urógena. Etiopatogenia y tratamiento quirúrgico y la defendió el 5 de diciembre de 1944 (Figuras 2 y 3). Tras prepararse en los más prestigiosos servicios de Urología españoles realizó un periplo de especialización urológica en el extranjero y, a su regreso, su vocación docente le anima a presentarse, en 1948, a la plaza vacante de la cátedra de Urología del doctorado en la Universidad de Madrid en la que compite, con sólo 29 años, con los profesores Alfonso de la Peña y Luis Cifuentes con quienes aprueba el primer ejercicio por encima de otros tres compañeros.

Retorna a la Universidad de Zaragoza y en 1950 desempeña el puesto de profesor ayudante de la cátedra de Patología y Clínica Quirúrgica con el Dr. D. Ricardo Lozano Blesa, hombre de extensa formación e intensa dedicación universitaria y con una 


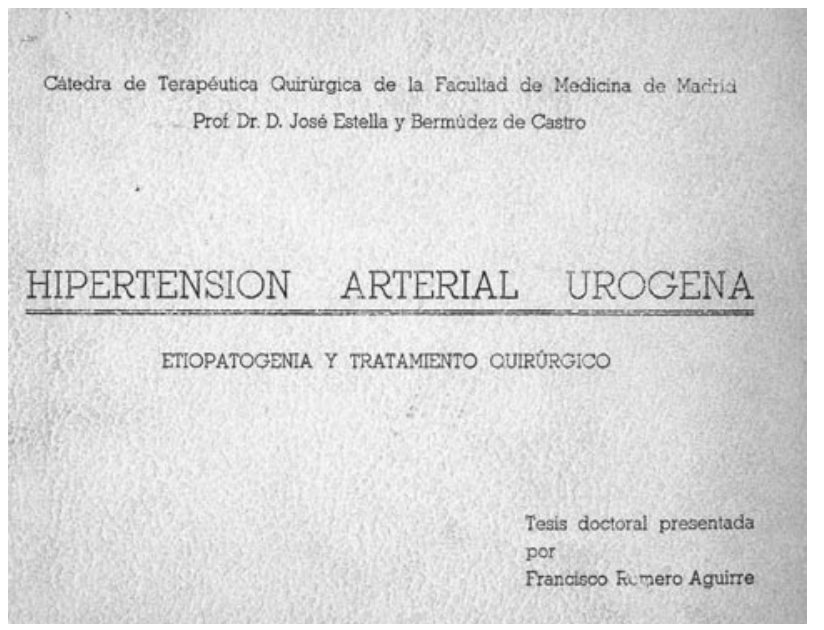

FIGURA 2. Portada de su memoria de tesis doctoral.

importante visión de futuro, quien aceptó desglosar del tronco común de la Cirugía las especialidades quirúrgicas y con cuyo apoyo y beneplácito se gestó la creación de la cátedra de Urología en el plan de estudios de la Licenciatura en la Universidad de Zaragoza.

El Decano de Medicina, D. Fernando Civeira Otermín, en la junta de Facultad celebrada el 29 de julio de 1954 (Figura 4), defiende la propuesta con los siguientes argumentos:

«La patología de las vías urinarias y la terapéutica quirúrgica del riñón vienen formando parte de las asignaturas "Patología Quirúrgica" y "Patología Médica". Sin embargo, es la realidad que esta parte de los conocimientos médicos ha alcanzado ya la suficiente independencia y personalidad para separarse didácticamente de las "Patologías Quirúrgica y Médicas" y constituir una asignatura independiente

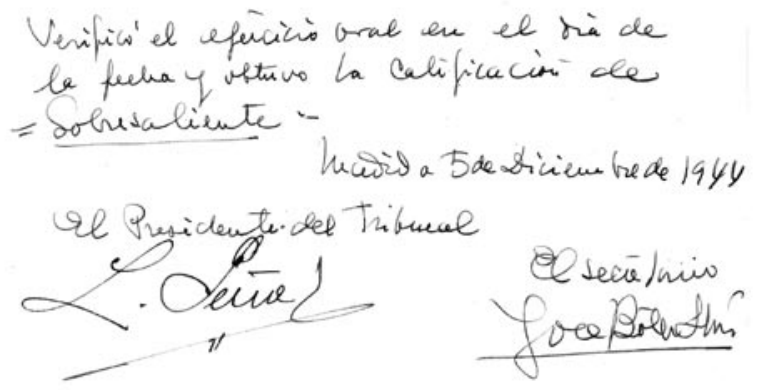

FIGURA 3. Calificación obtenida por su memoria de su tesis doctoral. como lo prueban la realidad del ejercicio profesional libre (los cirujanos generales no hacen urología y los urólogos no hacen cirugía general), de las publicaciones científicas (son distintas las revistas de Urología de las demás revistas médicas), la organización de los hospitales (en los que existe sistemáticamente un servicio de Urología), las reiteradas peticiones en este sentido de la Sociedad Española de Urología (resumidas en la petición que formularon a la última Asamblea de las Universidades Españolas), y sobretodo la realidad didáctica de nuestras Facultades en alguna de las cuales se ha separado ya como asignatura independiente contando con Profesor propio (Barcelona, Valencia, Madrid), llevan a esta Facultad, usando de la autorización que confiere el artículo 19 del vigente decreto ordenador del plan de nuestros estudios, a rogar a V. E. la autorice a:

Crear la Cátedra de "Patología de las Vías Urinarias y Terapéutica Quirúrgica del riñón (Urología)" y dar a esta nueva cátedra como cometidos específicos:

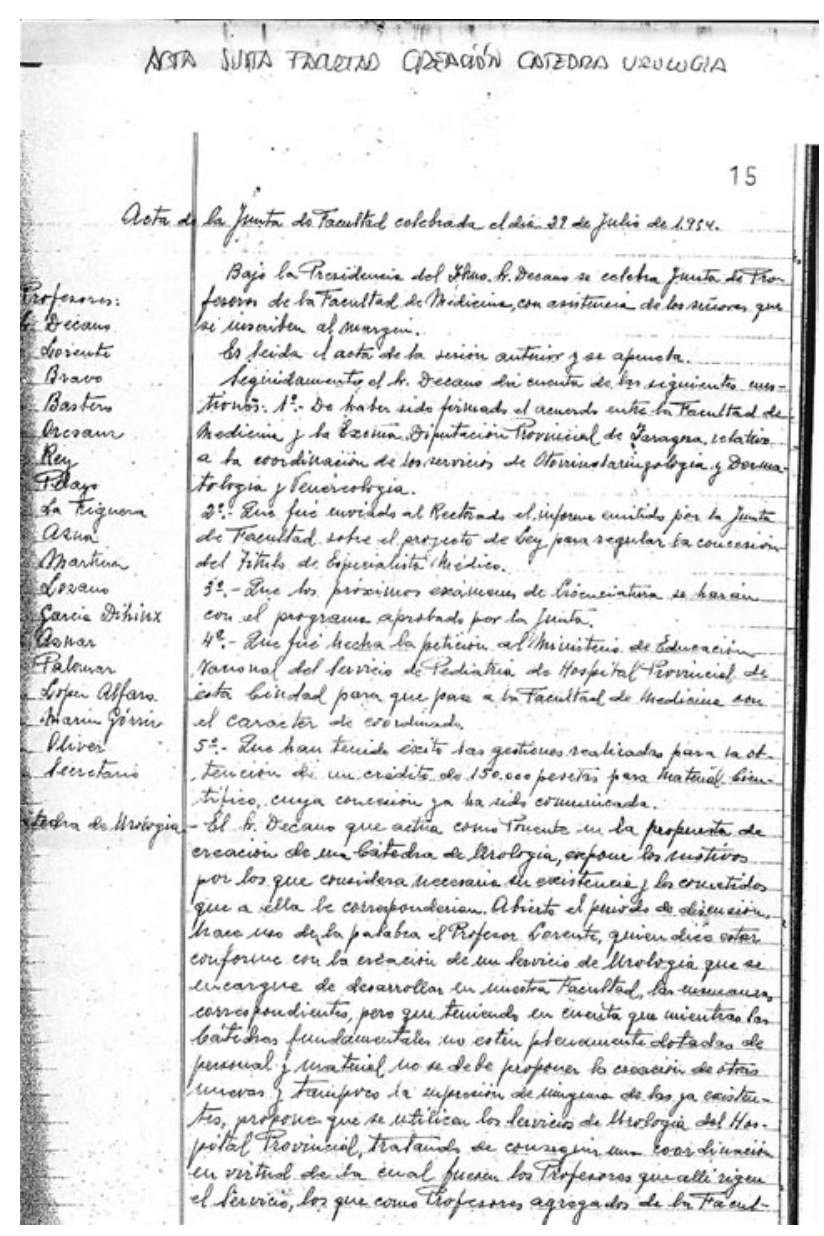

FIGURA 4. Acta de la Junta de la Facultad de Medicina en la que se aprobó la creación de la Cátedra de Urología, 1954. 
a) La enseñanza a los alumnos de la Licenciatura de Medicina de la Patología de las vías urinarias.

b) La preparación de especialistas en "Urología" (Patología de Vías Urinarias y Terapéutica Quirúrgica del riñón).

c) La prestación de la asistencia urológica a los enfermos que tengan la necesidad de ella en los servicios asistenciales de la Facultad de Medicina.

d) El investigar sobre sus materias específicas».

El claustro dio su apoyo a su creación con una votación favorable. De este modo se consolidó la constitución de la primera cátedra de Urología de la Universidad española que fue aprobada con carácter oficial por Orden Ministerial de 31 de enero de 1955 (BOE de 1 de marzo de 1955),

"Vista la propuesta de la Facultad de Medicina de Zaragoza, y de conformidad con lo establecido en el artículo 20 y en la disposición final del

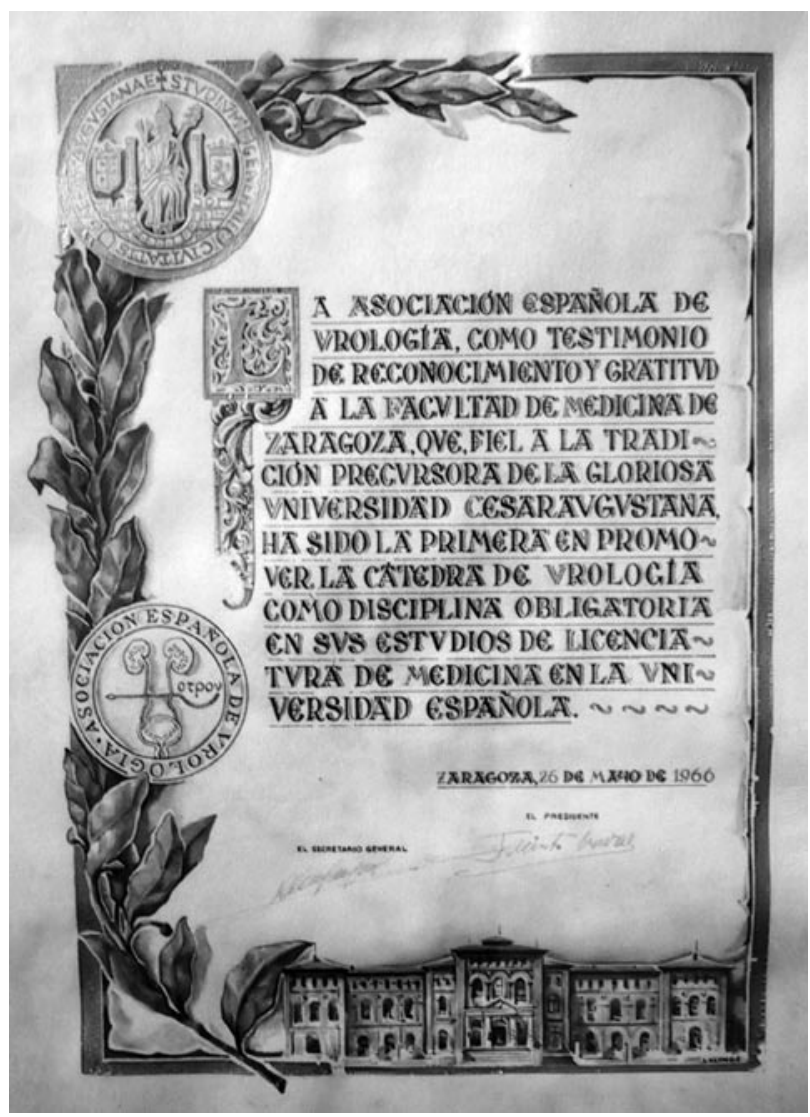

FIGURA 5. Diploma concedido por la AEU, 1966.
Decreto Ordenador de Estudios de la Facultades Universitarias de 11 de agosto de 1953, este ministerio ha resuelto que en el último curso de los estudios de la Licenciatura en Medicina en la expresada Facultad se curse la enseñanza de Urología».

En octubre de 1954 fue nombrado profesor encargado de la enseñanza de la Urología por acuerdo de la Facultad, y el año 1955 fue el primero en que se impartió la docencia urológica. A propuesta del Rectorado de Zaragoza y por resolución ministerial del 2 de enero de 1958, se creó una plaza de profesor adjunto en la Facultad de Medicina de la misma ciudad, adscrito a la enseñanza de la Urología y que, tras el correspondiente concurso, fue ganada por el Dr. Romero en septiembre de dicho año. En el BOE de 4 de octubre de 1960 se publicó la convocatoria para cubrir por oposición la cátedra de Urología y el 14 de diciembre de 1961 se desarrolló el concurso que aprobó con brillantez el que fue el primer catedrático numerario de Urología de la Licenciatura en la Universidad española, con lo que la especialidad recibió un importante impulso con su reconocimiento universitario.

En el transcurso del XXXI Congreso Nacional de Urología, celebrado en Zaragoza en 1966, la Junta Directiva de la Asociación Española hizo entrega a la Facultad de Medicina de la Universidad de Zaragoza, en un solemne acto académico, de un diploma en reconocimiento de haber sido la pionera en la enseñanza de la Urología como disciplina de la Licenciatura (Figura 5).

Su papel en la cátedra a partir de ese momento constituye una continua sucesión de actuaciones encaminadas a desempeñar su labor universitaria tanto docente como asistencial e investigadora, que comprende sus clases de la asignatura, los cursos del doctorado que impartía junto o alternos con los monográficos de Urología y la dirección de tesis doctorales a lo que sumó después la preparación de especialistas en la Escuela de Urología.

El programa del curso académico de la Licenciatura comprendía cuarenta y cinco lecciones que quedaron recogidas en un excelente manual, Urología Clínica, que fue editado por la cátedra en 1971 y 1974 en dos tomos, y refundido, ampliado y actualizado en un solo volumen en 1976, en 1977 y en una última en 1980; en 653 páginas compendia toda la patología de las vías urinarias y del aparato genital masculino además de reunir la virtud de tratarse, no solamente de un volumen dedicado a los estudiantes sino que también es de utilidad al médico general por su magnífica puesta al día de la materia. 


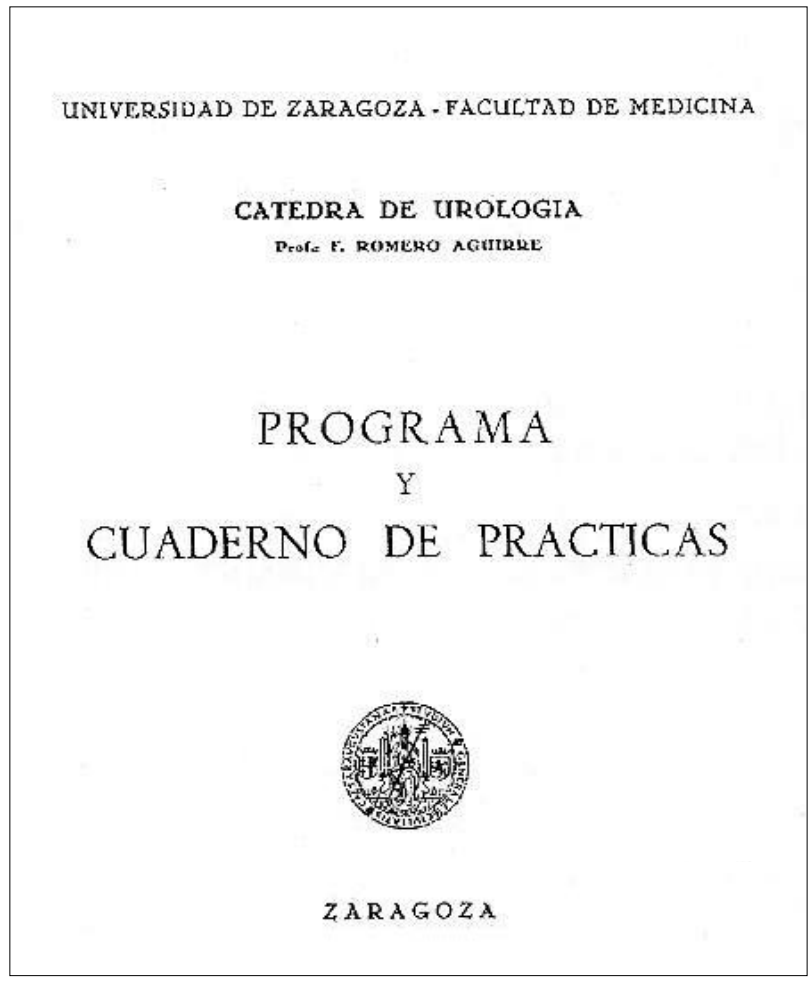

\section{FIGURA 6. Programa de prácticas, editado por la cátedra de Urología de Zaragoza.}

Contó el Dr. Romero en la cátedra con colaboradores a lo largo de los años en calidad, bien de ayudantes de clases prácticas (Figura 6), bien de médicos internos, bien de profesores adjuntos, interinos y por oposición, entre estos últimos Casimiro Romero Aguirre, durante tres años desde 1967 y, a partir de marzo de 1975, José Gabriel Valdivia Uría. Fue profesor encargado de curso Juan Escrivá Pla y como principal ayudante interino Jesús Valdés Buscarons.

Desde el año 1962, dictaba un curso del doctorado, que alcanzó gran prestigio por la categoría del profesorado y en el que participaban los miembros de la cátedra con el título de doctor, además de invitar a destacados compañeros de la especialidad, y al que asistían alumnos de todo el país (Figura 7). La investigación y la dirección de tesis doctorales fue otra de sus dedicaciones primordiales, desde 1969 y a lo largo de sus años de actividad universitaria fueron realizadas unas treinta tesis, aprobadas todas ellas con las máximas calificaciones (Tabla I).

Dirigió los cursos internacionales de Urología que se llevaron a cabo en la cátedra, en los que intervinieron profesores de gran prestigio nacional e internacional (Figura 8), llegó a alcanzar

\section{CURSO DEL CICLO DEL DOCTORADO SIMPOSIO DE CLAUSURA SOBRE ECOGRAFIA ENDOCAVITARIA EN UROLOGIA}

Facultad de Medicina.

Cátedra y Escuela Profesional de Urología (Prof. Francisco Romero Aguirre)

(Zaragoza , 15 de mayo de 1985)

Organizado por la Cátedra y Escuela Profesional de Urología de la Facultad de Medicina de Zaragoza y dirigido por F. Romero Aguirre y J. G. Valdivia Uría.

FIGURA 7. Programa del XXVIII curso monográfico del doctorado.

\section{Dr. Gose 9. Iglesias}

$$
\text { Mami, Fla., agosto 13, } 1969 .
$$

Prof. Don Frencisco Romero Aguirre, Avenida de la Lndpendencia l9,

Zaragoza,

Bspana.

Distinguldo Profosor y amigo:

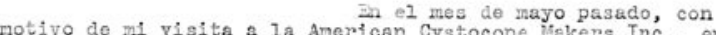
Now York bi saludándolo $y$ dendole las con Aurelio Uson, $y$ le escriespañoles por tocio lo que hicleron para lograr mi libertad.

Ho complazco en manifestaple, que tendré mucho gusté en colaborar cientificamente con Vás. Inclusive fo a Espana, toniendo la oportunidad de reiterar-

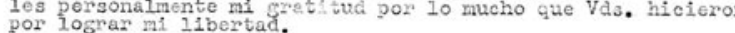
gestionos a gse rezpecto, ostuve bus bol teradas onterado por la lacoz

tunided de saludarlo personel Faporando poder toner la opor-

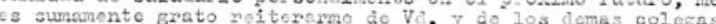
the ese pals, mis mas exprestves gracias por log favores reci bidos.

zo

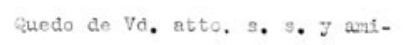

$$
\text { Dr. José Iglesias }
$$

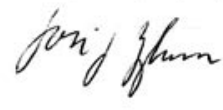

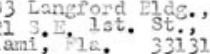

FIGURA 8. Carta de agradecimiento del profesor lglesias. 
la extraordinaria cifra de 500 matriculados, lo que nos da una idea de la aceptación que tenían. Del celebrado en abril de 1962 recogió el contenido de la exposición del plantel de especialistas, en una magnífica monografía Tumores de la Vejiga (Figura 9) (Tabla II).

XII-1969 Solé Balcells, Francisco Javier, Fisiopatología de la obstrucción urinaria en la infancia.

XII-1969 Gosalvez Jordá, Rafael, Estudio radiológico de la micción en el niño mediante la CUMS.

X-1970 Guelbenzu Morte, Francisco J., Trasformación del aparato yuxtaglomerular del riñón en diversas circunstancias de isquemia renal aguda.

I-1971 Sarmiento García, Guillermo, Experiencias clínicas en el litotritor eléctrico Urat-1.

II-1973 Valdivia Uría, José Gabriel, Uroflujometría. Aportaciones a la técnica y a su valor diagnóstico.

II-1973 Rioja Sanz, Luis Ángel, Aportación a las técnicas de reimplantación uretero-vesicales.

X-1973 Ollé Carreras, José, Hidatidosis renal.

XI-1973 Sánchez Salvador, Andrés, Cáncer infiltrante de vejiga urinaria.

XI-1974 Aguilera Ramos, Carlos, Aportación al estudio Urodinámico de las obstrucciones cérvico prostáticas.

XII-1976 Romero Fernández, Francisco Javier, Investigación bacteriológica sobre Urolitiasis., tejidos uroandrológicos e infección urinaria.

IV-1977 Liédana Torres, José Miguel, Aportación al diagnóstico de las afecciones prostáticas con diversos métodos de biopsia.

VI-1977 López López, José Antonio, Aportación al estudio de la patología expansiva renal con ultrasonidos.

XI-1977 Rioja Sanz, Carlos, Aportación al estudio urodinámico de la incontinencia de orina en la mujer.

I-1978 Sanz Guajardo, Dámaso, Hialinosis focal glomerular. Correlación clínico patológica.

VI-1979 Bayo Ochoa, Ángel, Aportación al diagnóstico y tratamiento de la infertilidad en el varón.

1980 Sánchez Chapado, Manuel Vicente, El fenómeno enurético. Estudio experimental en Aragón.

1982 Elizalda Amatria, Ángel Gabriel, Citodiagnóstico de la patología testicular por punción aspiración con aguja fina.

1983 Franco Manzano, Ricardo, Aportación al estudio del lugar de formación de los productos de degradación del fibrinógeno/fibrina en el tracto urinario y su relación con la patología urológica.

1984 Escrivá Plan, Juan, Nuevos métodos cistométricos en Urología.

1985 Pomerol Serra, José María, La esterilidad masculina.

Aranda del Villar, José M., Urolitiasis en el distrito universitario de Zaragoza. Morbilidad y características.

Parés Guillén, Joaquín, Linfografía en Urología.

Valdés Buscarons, Jesús, Estudio de las funciones renales de la hipertensión.

Moral Nadal, Juan Ignacio, Aportación al estudio urodinámico de los traumatismos medulares.

Villarroya Rodríguez, Serafín, Aportación al estudio experimental ultraestructural y funcional del riñón con hipertrofia compensadora tras distintos casos de ablación parenquimatosa.

Sanz Vela, José Ignacio, Aportación al estudio del síndrome miccional en la mujer.

Caro Cebrián, Carlos J., Aportación al tratamiento farmacológico de las disfunciones vésico esfinterianas.

Nogueras Jimeno, Miguel Ángel, Aportación al estudio de las infecciones en cirugía urológica endoscópica.

Otero Mauricio, Gerardo, Aportación al estudio de la génesis de los productos de degradación del fibrinógeno-fibrina en infecciones urológicas. 


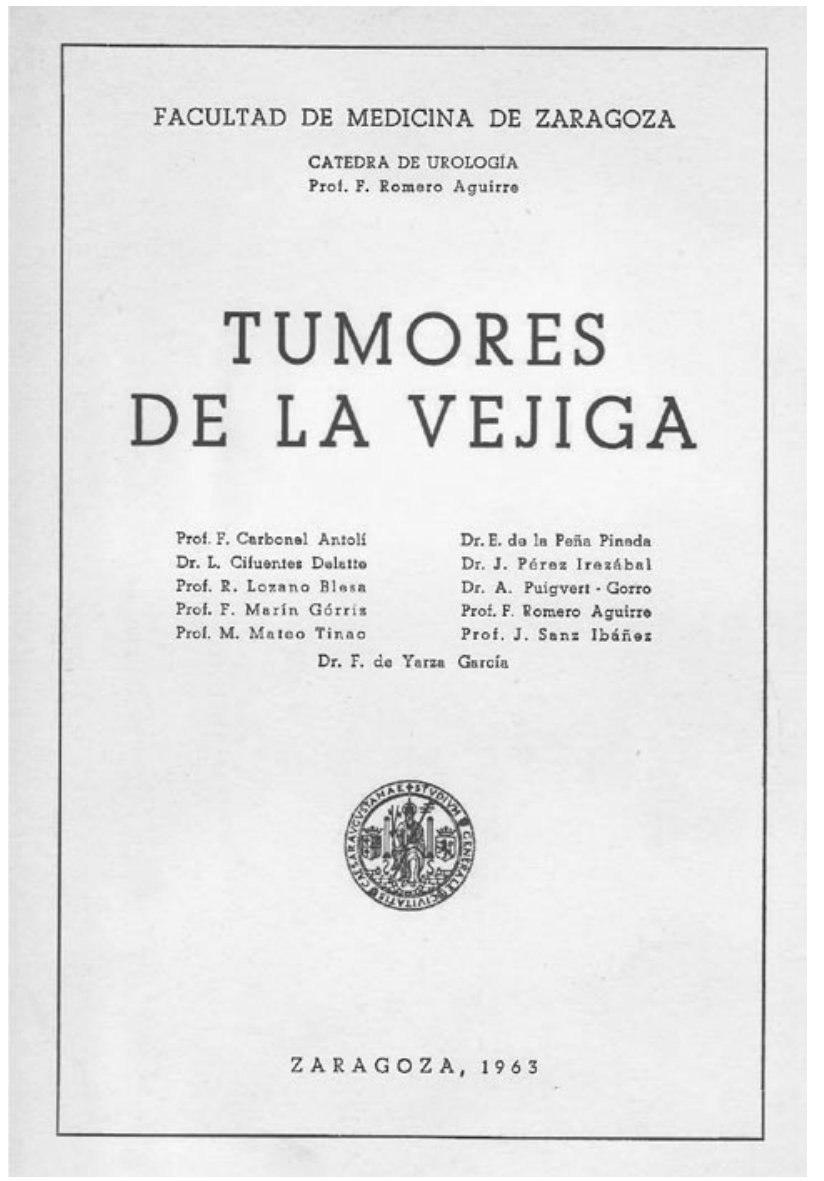

FIGURA 9. Curso Internacional de Urología Tumores Vesicales, 1963.
Uno de sus mayores logros fue la creación de la Escuela Profesional de Urología de Zaragoza, dependiente de la Cátedra de Urología de la Facultad de Medicina de la Universidad y de la que se considera como un órgano de extensión, de acuerdo con la Ley de Ordenación Universitaria. Sus estatutos fueron aprobados por Orden Ministerial del día 19 de noviembre de 1969 (B. O. de 19 de mayo de 1970), [recogidos en Arch. Esp. Uro. XXIII (4): 413 $416]$.

"Vistos los favorables informes y dictamen del Rectorado de la Universidad de Zaragoza y del Consejo Nacional de Educación, respectivamente; y de acuerdo con el artículo 23 de la Ley de Ordenación Universitaria de 29 de julio de 1943, este Ministerio ha resuelto: Crear la Escuela Profesional de Urología adscrita a la Cátedra de Urología de la Facultad de Medicina de Zaragoza y aprobar sus estatutos por los que se regirá la mencionada escuela».

Sus objetivos eran la formación de los médicos que aspirasen a ejercer la especialidad y el perfeccionamiento de los ya capacitados, además de fomentar la investigación, facilitar la realización de tesis doctorales y promover el intercambio científico con instituciones afines nacionales y extranjeras. Para cumplir estas funciones contaba con las instalaciones y el material de la cátedra de Urología en la Clínica universitaria de la Facultad de Medicina

1962 Tumores de la vejiga.

1967 Progresos en Urología.

1968 Patología del cuello vesical y de la próstata.

1970 Avances nefro-urológicos.

1971 Esterilidad masculina.

1975 Urolitiasis.

1977 Temas de actualidad de las neoplasias urológicas.

1978 Progresos en Urología.

1979 Temas de actualidad en Urología.

1980 Endoscopia urológica y temas de actualidad en Urología.

1981 Trasplante renal y cirugía vásculo-renal experimental.

1882 Temas actuales en Urología.

1983 Endoscopia urinaria y Avances en Urología.

1984 Actualizaciones urológicas.

1985 Temas actuales de Urología y Ecografía endocavitaria en Urología.

1986 Cirugía urológica actual y Docencia en Urología (curso XXV aniversario).

1987 Endoscopia urinaria. Curso homenaje, el último en el que participó. 
y del servicio de Urología del Hospital Provincial de Nuestra Señora de Gracia, de Zaragoza, además de la colaboración de los centros asistenciales donde prestaba sus servicios el profesorado (Figura 10).

El plan de estudios comprendía la especialización de postgrado en Urología, con cinco alumnos por año y un periodo de duración de dos cursos, con una parte teórica que incluía un programa completo de conocimiento de la materia y otra práctica que se llevaba a cabo por la asistencia obligatoria del alumno, diariamente y por espacio de cinco horas, a los servicios clínicos en los que rotaba por la consulta, por el laboratorio, por los departamento de radiología, de endoscopia y de uromanometría, además de acudir a la planta de enfermos hospitalizados, al quirófano y a la sala de cirugía experimental. Incluía también la realización de guardias en las urgencias urológicas y el tomar parte progresivamente en todas las actividades de la Clínica, con el deber de participar en los seminarios, sesiones científicas, cursos monográficos de perfeccionamiento y de ampliación de estudios que se dictasen además de efectuar una

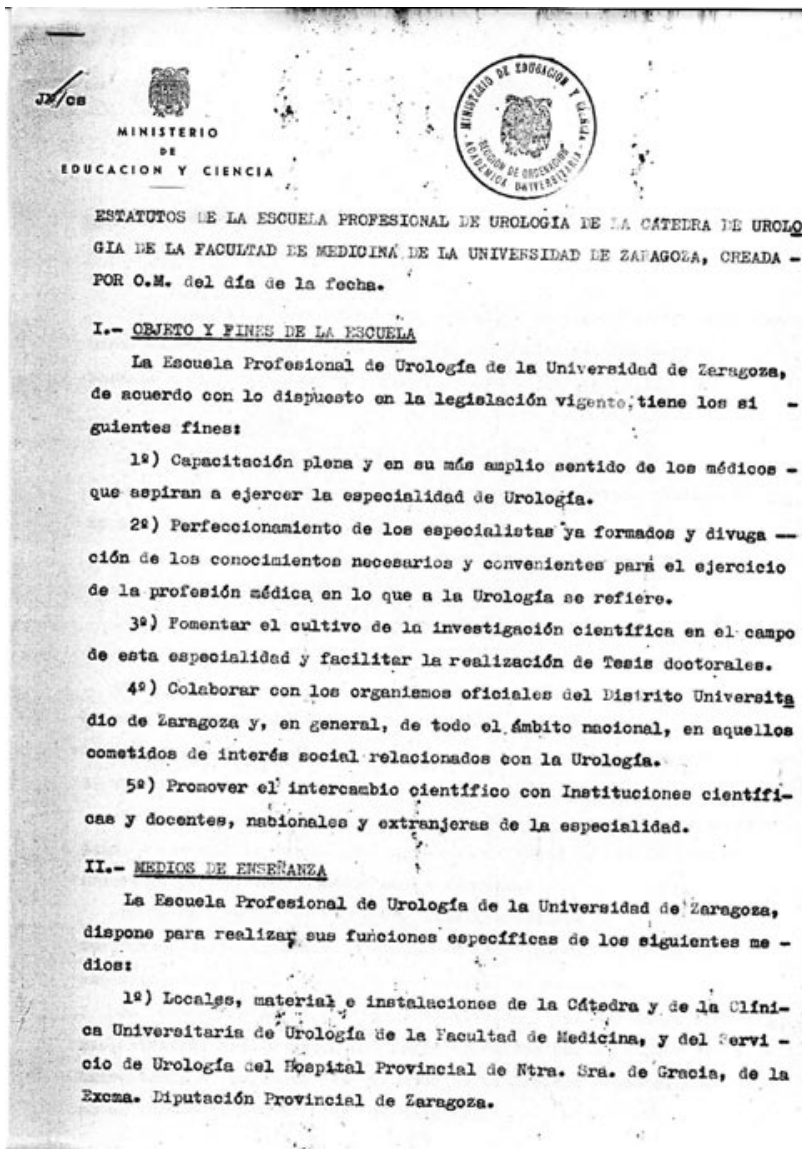

FIGURA 10. Estatutos de la Escuela Profesional de Urología de la Universidad de Zaragoza, 1970.

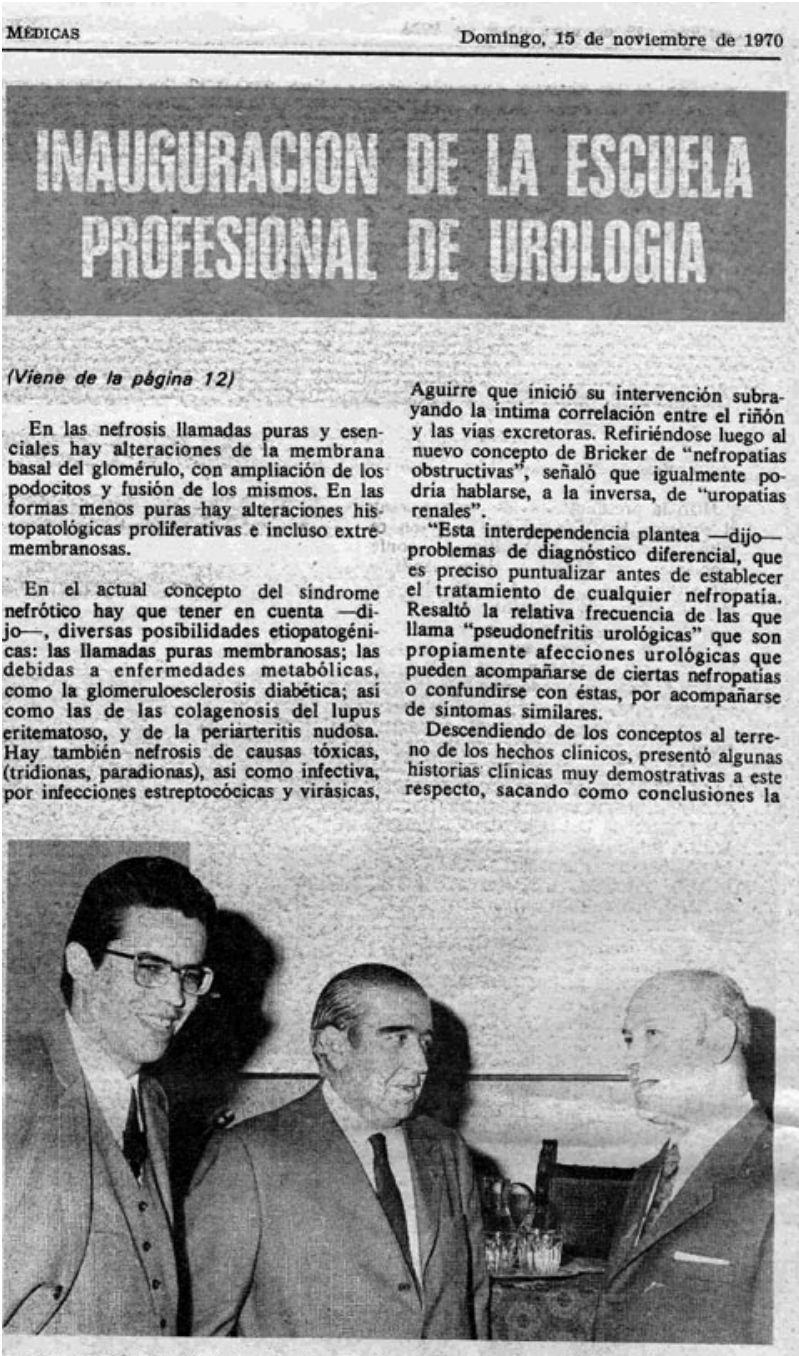

El profesor Romero Aguirre con los doctores Yarza Garcia (centro) y Valdivia Uria.

FIGURA 1 1. Acto inaugural de la Escuela profesional de Urología de Zaragoza, 1970.

memoria que contuviese una parte de investigación de acuerdo con la metodología científica.

Al terminar cada curso preceptivamente se efectuaba una evaluación consistente en el desarrollo escrito de dos temas, un ejercicio práctico y el comentario y análisis del trabajo científico realizado para obtener, con el aprobado de los dos cursos académicos, el título oficial de especialistas en Urología. Salían bien formados, con un amplio bagaje de conocimientos teóricos y prácticos en la exploración clínica y en la quirúrgica, además de la preparación en la actividad de experimentación para el desarrollo de tesis y de su exposición pública en congresos y reuniones (Tabla III).

En el acto inaugural de la escuela (Figura 11), la quinta oficialmente reconocida en el país, el 27 de octubre de 1970, agradeció los apoyos recibidos, 
TABLA III.

1971 Gonzalo Arruelo Tomás

Ángel Cuesta Climent

Leopoldo Klee Palacio

Emilio García Martínez

1972 Ángel Bayo Ochoa

1974 Jesús María Salanueva Ferrer

Fernando Chu Tham

Tanios Iskandar Karma

1975 Serafín Villarroya Rodríguez

Juan Severa Muntaner

1976 Francisco Javier Romero Fernández

José Antonio López López

Ángel Elizalde Amatria

Miguel Rivera Morocho
1978 Yolanda Mengod Guillén

Alberto Christian Barros Hernández

José Gómez Utrera

Joaquín Taberner Llop

Adel Mohamed Abou Yah-Yah

1979 Ausberto José Daza Luque

Haidar Hassan Ajami

Luis Miguel Flavian Domenech

1980 Manuel Martín Montañés

Juan Mora Nadal

Manuel Sánchez Chapado

Manuel Ruiz Ramo expresó su satisfacción por su creación y ofreció la escuela a toda la juventud deseosa de adquirir formación urológica en beneficio del enfermo, objeto principal de la enseñanza médica. Le auguraba un esperanzador porvenir, no por sus limitadas posibilidades personales, sino por la inestimable ayuda de todos sus colaboradores y por el amparo ofrecido por todos los estamentos universitarios y provinciales.

El XXV aniversario de la creación de la cátedra, celebrado en 1980, reunió a cuantos habían participado en ella desde 1954 y a los especialista allí formados, lo que constituyó un merecido reconocimiento de su gran labor docente, recogida en una memoria del acto (Figura 12).

A lo largo de toda su vida académica desempeñó importantes cargos académicos de responsabilidad en la Facultad de Medicina, fue vicedecano en

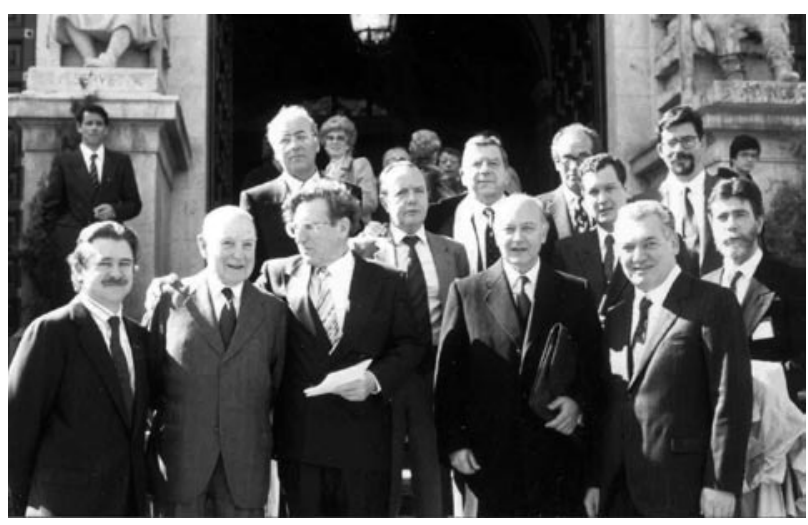

FIGURA 12. Conmemoración del XXV aniversario de la creación de la Cátedra de Urología, 1980.
1970 y decano entre 1973 y 76, años en los que desarrolló una gran actividad, en los que se produjo el traslado de la Facultad al campus universitario de San Francisco y en los que, en febrero de 1975, se inauguró el nuevo Hospital Clínico de Zaragoza. En 1976 fue nombrado director del departamento de Cirugía de la Facultad. Con motivo de su jubilación reglamentaria, el 1 de octubre de 1986, tuvo lugar un curso extraordinario que constituyó un emotivo homenaje de todas las escuelas de la Urología españolas a su labor universitaria.

\section{BIBLIOGRAFÍA Y LECTURAS RECOMENDADAS ( lectura fundamental)}

1. INSAUSTI CORDÓN, J.L.: "Compendio histórico de la Urología española y de su Asociación". Ponencia IV Congreso Iberoamericano de Urología, Acapulco (Méjico), pág. 70, Reicosa, Madrid, 1982.

2. LOVACO CASTELLANO, J.M.; GARCÍA CUERPO, E.; LOVACO CASTELLANO, F.: "La Urología en Aragón". Historia Biográfica y Bibliográfica de la Urología Española en el siglo XX. Edicomplet, Madrid, 2002.

*3. PÉREZ AlbACETE, M.: "Cien Figuras de la Urología Española”. Pág. 196. Tip. San Francisco, Murcia, 2005.

**4. ROMERO FERNÁNDEZ, F.; VALDIVIA URÍA, G.; RIOJA SANZ, L.: "El profesor Romero Aguirre, Catedrático de Urología de la Universidad de Zaragoza (1961-1986)". Urol. Integr. Invest., 7: 226, 2002. 